JOINT COMMISSION II

Automated Telescopes for Photometry \& Imaging Performance \& Results with IR Arrays

Commission 25 with 9 Chairman \& Editor: I.S. McLean 


\title{
AUTOMATED TELESCOPES FOR PHOTOMETRY AND IMAGING: PERFORMANCE AND RESULTS WITH INFRARED ARRAYS
}

\author{
IAN S. MCLEAN \\ Depts. Astronomy \& Physics \\ UCLA, \\ 8979 MSA, \\ Los Angeles, CA 90024-1562 \\ USA
}

\begin{abstract}
An edited review of the presentations given at Joint Commission Meeting II during the 21 st General Assembly of the IAU is presented.
\end{abstract}

\section{Introduction}

The unusual format of this Joint Commission Meeting arose when Commissions 25 and 9 proposed to hold joint sessions to discuss two exciting and important topics in observational astronomy, namely the development of fully automated telescopes and the rapid growth of infrared astronomy stimulated by the advent of infrared array detectors. The IAU Executive Committee suggested to myself (as President of Commission 25) and Prof. John Davies (President of Commission 9) that both topics would be worthy of a JCM if we could combine them into one unit. Although the time for each topic would be only 3 hours, we agreed with the idea because of the significance of both fields. Separate organising committees were formed for each part of the JCM. The morning session was allocated to Automated Telescopes for Photometry and Imaging and was ably organised and chaired by Dr. Saul Adelman to whom I am most grateful. The afternoon session covered Performance and Results with Infrared Arrays and was organised by my colleague Prof. Eric E. Becklin and myself; the session was chaired by the author. Both meetings were well attended. Since time was short, speakers were asked to concentrate on highlights. This paper provides an edited summary of the presentations given.

\section{Automated Telescopes for Photometry and Imaging}

In the last decade a few automated telescopes were built for especially repetitious observations such as filter photometry and CCD imaging. Now other astronomers are planning and /or building additional automated telescopes typically in the $1-\mathrm{m}$ class. These telescopes can be linked into a global network to coordinate observations and to obtain long time coverage. This 3 hour Joint Meeting considered how automated telescopes are changing the science being produced. 


\subsection{LIST OF PAPERS}

1. David L. Crawford, Kitt Peak National Observatory: "The Advent of a Global Network of Automated Telescopes".

2. Andrew T. Young, San Diego State University: "Precision Photometry".

3. Francois R. Querci and Monique Querci, Observatoire Midi-Pyrenees: "High Precision Photometry; Project of Automated Stations With Three 1-m Telescopes".

4. Kwan-Yu Chen and Frank Bradshaw Wood, University of Florida; Shi-yang Jiang and Ji-tong Zhang, Beijing Astronomical Observatory, Academia Sinica; Pei-sheng Chen and Yu-lan Yang, Yunnan Observatory, Academia Sinica: "The 0.4-m South Pole Optical Telescope Project".

5. Chris Sterken, University of Brussels and J. Manfroid, Universite de Liege: "Robotic Photometry and Precision: Our Experiences Over Three Years".

6. E. F. Milone, T. A. Clark, and J. T. Himer, University of Calgary: "Infrared Variable Star Observing from the Rothney Astrophysical Observatory".

7. Edward F. Guinan, Villanova Unversity: "Report from the Four College APT Consortium".

8. Marcello Rodino, Catania Astrophysical Observatory: "The $0.8 \mathrm{~m}$ APT at Catania Astrophysical Observatory".

9. M. Loudon, J. Priestley, and Edwin Budding, Carter Observatory: "A Low Cost Prototype APT Working in the Southern Hemisphere".

10. Terry Oswalt, Florida Institute of Technology: "The SARA Kitt Peak 0.9-m Telescope Project".

11. A. G. Davis Philip, Wesleyan University and Union College: "Some Thoughts on An Automated Imaging Telescope".

12. Saul J. Adelman, The Citadel: "Automated Spectrophotometry".

\subsection{SUMMARY OF PAPERS}

Crawford began the session by presenting the concept of a Global Network of Automatic Small Telescopes (GNAT) as an idea whose time is right, scientifically and technically. This concept should be implemented in the not too distant future. The only question is how and when. It is important that it be done in a reasonably planned way so as to take advantage of the many potentials. He welcomes any and all comments and critiques. Such input will be used to help formulate one or more proposals for funding in an effort to bring the concept of a GNAT to reality.

Young was concerned with the question: Why is photometry the worst of all physical measurements? We do not know what we are measuring; and we do not measure what we need. He suggested ways to place astronomical photometry on a scientific basis. His message that we need to have much higher precision photometry was concurred by a number of speakers. With the concept of a Global Network of Automated Small Telescopes, it would be a shame not to use the GNAT to promote the goal of having many telescopes on the same photometric system.

F. Querci reported that fifty French and Moroccan scientists are investigating an automated photometric telescope station involving three 1-m telescopes for differential photometry of variable stars. The first station may be set-up in Oukaimeden, Morocco. This proposal is based on tests performed with a prototype of two C-11 telescopes at the Observatoire de Haute-Provence. This group is investigating additional sites in the Northern Saharian region.

Wood, speaking for his colleagues, discussed a $40-\mathrm{cm}$ reflector designed especially for automated operation at the South Pole. It is being constructed for continuous photometry of 
variable stars. The South Pole is an attractive and unique location for an optical telescope for astronomical observations because of its high altitude, very low atmospheric water vapor content, and the long duration of continuous darkness. In 1986 the first program of stellar photometry at this location was carried out with the operation of an automatic optical telescope which is a two-mirror siderosat with an 8-cm lens and photoelectric photometer. The initial observations from the 8-cm telescope concentrated on observing the bright Wolf-Rayet binary system 2 Velorum. Preliminary results indicates that the extinction at the South Pole is similar to those at other isolated high altitude observatory sites, and sky conditions allow long continuous observations to take place. The completion and accomplishment of the work with the 8-cm South Pole Optical Telescope naturally lead to planning of a larger telescope. A joint project by a group of Chinese and American astronomers commenced in 1986. The aim of the project is to provide a facility for continuous photometric observations of selected variable stars.

Sterken discussed the experiences that he and Manfroid have had with the same photometric telescope, the Stromgren Automatic Telescope located at the European Southem Observatory at La Silla, Chile, both before and after upgrading to an automatic configuration. The major lessions that they learned are: 1) Automated telescopes are only as good as the software that runs them. The programming language must be highly sophisticated to allow for very flexible operation during the observations. 2) A good programming language is not enough. The command files written by the user should be complete and well-tested. 3) Refurbishing an old telescope for automatic operation may be comparable in cost to that of building or buying a very compact specifically designed photometric telescope. 4) To improve on the accuray a faster instrument both mechanically and optically is needed.

Milone reviewed infrared variable star observing from the Rothney Astrophysical Observatory in Calgary, Canada. The metal mirror will soon be replaced by a high quality optical mirror. This telescope works in a semi-automated mode.

Guianan discussed the participation of the Astronomy Department at Villianova University in the Four College APT Consortium. The other schools are The College of Charleston, The Citadel, and the University of Nevada - Las Vegas. The principal astronomer is Dr. Robert J. Dukes, Jr. of the College of Charleston. This consortium operates a 30-inch telescope on Mt. Hopkins, Arizona. The projects undertaken include observations of solar type stars, multi-mode variable stars, chemically peculiar stars of the upper main sequence, apsidal motion of eclipsing binaries and Be stars.

A major project at Villanova is entitled "Sun in Time" Project. It is a coordinated optical APT, UV, and X-ray study of G0 V to G5 V proxies for the Sun from its arrival on the ZAMS (70Myr) to the present. Guinan, G. McCook (Villanova), J. D. Dorren (Univ. of Pa.) and Villanova undergraduates are studying the evolution of magnetic activity of the Sun in time using the IUE satellite, ROSAT, and ground-based photometry with the APT. They have selected several single G0 V to G5 V stars as proxies for the Sun at different ages in its main-sequence lifetime. Following contraction to the ZAMS, the young Sun is expected to have been rotating much more rapidly than today, with magnetic braking subsequently producing a spindown to its present 25.4 day rotation period. The young Sun's more rapid rotation and, consequently, stronger magnetic dynamo is expected to result in more vigorous magnetic activity from the photosphere, chromosphere, transition region, and corona, as well as an enhanced solar wind. The stars selected for study include relatively bright nearby G0-G5 V stars whose ages are determined from membership in moving groups. In addition, observations are being made of the solar twins--HD44594, 16 Cyg A, and 16 Cyg B. These stars are among the closest known matches to the present Sun and are being studied as additional examples of mid-life G2 V stars. Photoelectric 
photometry of all the stars except HD44594 (which is too far south) is being conducted with automatic photoelectric telescopes (APTs) at Mt. Hopkins, AZ. The photometry is being carried out to monitor starspot activity discovered on these stars. Rotation periods and the percentage of the stars' surfaces covered with spots are being directly determined from the APT photometry.

Rodino reported that the $0.8 \mathrm{~m}$ APT purchased from Autoscope by the Catania Astrophysical Observatory should begin operations in the next few months. Their site is on the slope of Mt. Etna in Scilily.

Budding reviewed his efforts with $\mathbf{M}$. Loudon and J. Priestley to covert an "Celestron Compustar" into an automatic photoelectric telescope. He discussed the background and development of this operation along with some practical limitations of the current arrangement and their causes. Some typical preliminary photoelectric data were presented.

Oswalt reported for his colleagues that the Southeastem Association for Research in Astronomy (SARA), a consortium of the Florida Institute of Technology, East Tenessesse State University, University of Georgia and Valdosta State College, is recommissioning the former No. $10.9-\mathrm{m}$ telescope at Kitt Peak National Observatory as a fully-automated facility for CCD imaging and photometry at a new site on Kitt Peak. In its role as the primary astronomical research instrument for the SARA universities and the first fully automated general purpose telescope at Kitt Peak, this instrument will support a long-term science program involving well over a dozen proposed research projects.

Philip for himself and Hayes noted that automatic photoelectric telescopes are now in operation at least 8 sites over the world performing photoelectric measures of stellar magnitudes. Larger, new telescopes are being planned and built. The next step in using automatic telescopes in his opinion is to apply CCD techniques and allow CCD four-color photometry to be done for hundreds of stars at a time. Such a system could embark on some major,long-term projects and provide many astronomers with large amounts of astronomical data to analyze. These data can be transformed to astrophysical parameters and be used, for example, to study stars in late stages of stellar evolution.

Adelman stated that several important astrophysical problems require new instrumentation to obtain higher quality spectrophotometric data. An Automated Spectrophotometric Telescope with a modern array detector should have at least the accuracy of a photomultiplier scanner, be able to simultaneously record the fluxes in many bandpasses, and produce data that compares with the best photometry in quality. Recent progress with Automated Photoelectric Telescopes indicates that automated telescopes with apertures of $0.75-\mathrm{m}$ and larger are suitable platforms for spectrophotometric instrumentation. Several instrumental designs are being considered for automated operations. The reduction of spectrophotometric data and the operation of Automated Spectrophotometric Telescopes were discussed.

\section{Performance and Results with Infrared Arrays}

In marked contrast to optical astronomy and radio astronomy, the main limitation to the growth of infrared astronomy has been detector technology. No longer is this the case. Now, with the advent of infrared "arrays" --- small solid-state imaging devices with 2-dimensional (x-y) arrays of tens of thousands of infrared-sensitive picture elements --- measurements deemed quite impossible using the old style single-element detectors and scanned apertures are not only viable, they are surprisingly competitive in depth and photometric precision with optical CCD techniques. 
Considerable experience has now been gained in performing photometry and polarimetry with these arrays and the results are very encouraging. Below is a list of the papers presented at this meeting followed by an edited summary of each.

\subsection{LSIT OF PAPERS}

\section{A. INSTRUMENTS AND DETECTORS:}

1. "Astronomy with infrared array detectors": Ian S. McLean, University of Califomia, Los Angeles.

2. "Infrared array instruments at NOAO and CTIO": Brooke Gregory, Cerro Tololo Inter-american Observatory.

3. "Performance of HgCdTe arrays --- ground-based and HST": Rodger Thompson, Steward Observatory, University of Arizona

4. "Early results from IRIS": Peter R. Gillingham and David A. Allen, Anglo-Australian Observatory.

5. "IR developments in France": J. L. Monin, Observatoire de Grenoble

6. "ISOCAM flight model tests": Diego Cesarsky, France.

B. ASTRONOMY WITH INFRARED ARRAYS:

7. "Experience with InSb and IBC arrays; SIRTF and ground-based": Judith Pipher, University of Rochester.

8. "Applications of photometry with infrared arrays to globular cluster CMD studies": Andy Longmore and C. Buckley, Royal Observatory, Edinburgh.

9. "Sub-arcsecond IR observations with CIRCUS and diffraction-limited IR observations with COME ON": F. Rigaut

10. "High spatial resolution images of the NIR continuum and HeI line emission of the

Galactic Center": Andreas Eckart and Reinhard Genzel, Max Planck Institue, Munich.

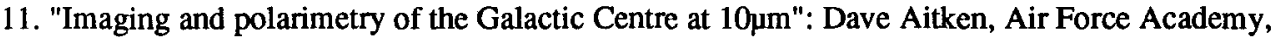
Australia.

12. "High-background imaging: results from 5-18 $\mu \mathrm{m}$ array camera program": Dan Gezari, Goddard Space Flight Center.

\subsection{SUMMARY OF PAPERS}

Mclean began the session with a general overview. He described an exciting "decade of development" from which it was clear that Infrared Astronomy is a fast-changing subject! In the early eighties a few astronomy groups were bold enough to explore the (very few) "array" detectors then available. Typical formats were $1 \times 32$ or $32 \times 32$ pixels. In 1986 a generation of new, high quality infrared array detectors came onto the scene. These devices had approximately $64 \times 64$ pixels. One device, made from indium antimonide (InSb), had $62 \times 58$ pixels and was sensitive (70\% quantum efficiency) from $1-5 \mu \mathrm{m}$ in wavelength when operated at $35 \mathrm{~K}$. The IRCAM infrared camera on UKIRT is typical of the several InSb array instruments now in use and for which a considerable experience has been amassed concerning their photometric properties (McLean 1987, McLean etal. 1989). Detectors made from the material mercury-cadmium-telluride (HgCdTe) appeared around the same time. Those arrays were tailored to work at the higher temperature of $77 \mathrm{~K}$ by limiting their band-gap to correspond to a shorter wavelength interval from $1-2.5 \mu \mathrm{m}$. Although initially $64 \times 64$, within a few years the $\mathrm{HgCdTe}$ detectors achieved formats of $128 \times 128$ 
and also succeeded in improving noise performance. Several instruments employing $128 \times 128$ $\mathrm{HgCdTe}$ arrays are now in use. Arrays for longer infrared wavelengths have lagged behind, but not by much.

Already the near- and mid- infrared technologies have reached $256 \times 256$ pixels and some preliminary astronomical results from these new detectors will be discussed at this meeting. Also the technology of platinum silicide has been used successfully in astronomy. Unfortunately, these detectors have a very low quantum efficiency (a few \%) which is partially compensated by low noise, large format --- due to the all-silicon technology --- and excellent cosmetic quality. Devices with $256 \times 256$ pixels are in use at the Kitt Peak Observatory and a very large PtSi array (640x486 pixels) made by the Kodak company has been used by McCaughrean, Angel and Mentzell for astronomical imaging of bright sources (McCaughrean 1991).

Just as significant as the rate of growth of the size and sensitivity of infrared arrays has been the astronomical achievements of astronomers pushing the new devices to their limits (see the proceedings of the conference held in Tucson, Arizona in February 1990; Elston 1991). Naturally the new infrared arrays have found immediate application in cameras for a wide range of morphological studies. Incredibly sharp and detailed infrared images, many presented as "three-colour composites", are now available for many classes of cosmic sources from planets, to star-forming regions, from the Galactic Center to distant extragalactic sources. These infrared pictures have had a profound effect on the subject. Imaging at infrared wavelengths is vital for many important survey projects, such as the search for cool, low-mass stars and brown dwarfs whose optical emission is weak, or searches for highly obscured sources heavily reddened by dust, or surveys of galaxies at high redshifts. But that is not all.

Infrared arrays are proving to be quantitative, photometric tools. For example, excellent new colour-magnitude diagrams of globular clusters, open clusters and star-forming regions have been obtained; luminosity functions have been derived; galaxy light profiles to very faint levels; infrared photometry and polarimetry of numerous galactic regions including the Galactic Center; and extremely deep $2.2 \mu \mathrm{m}$ infrared imaging photometry of galaxies at high redshifts. The latter is a consequence of the stability and extremely good flat-field performance of these arrays so far -.- better than 1 part in $10^{5}$..- leading to limiting magnitudes of $\mathrm{K}(2.2 \mu \mathrm{m})=24$ in a very long background-limited exposure (Cowie etal. 1990).

Moreover, the wavelength range benefitting from these array detectors has not been limited to the near infrared thanks to the availability of arrays of doped silicon photo-conductors such as the $62 \times 58 \mathrm{Si}: \mathrm{Ga}$ array from SBRC, or the new Impurity Band Conduction (IBC) devices being developed for low-background space astronomy. Of course, for ground-based infrared astronomy at these wavelengths the "background" thermal emission from the telescope and the sky is a serious limitation which results in the need for very high speed readout of the array to avoid saturation.

High speed infrared imaging is also extremely valuable in obtaining high spatial (angular) resolution. Seeing conditions are generally observed to be better at infrared wavelengths. The technique of "slow-speckle" imaging can yield near diffraction limited performance.

With the continuing improvement in the performance of infrared detectors, in particular lower readout noise and lower dark currents, it is already feasible to employ them in infrared spectrometers. The new generation of infrared spectrometers are almost as large as optical spectrographs but with the added complication that the grating (and everything else) must be cooled to cryogenic temperatures $(77 \mathrm{~K})$ to eliminate unwanted thermal emission. For studies of galaxies at high redshifts infrared spectrometers such as the CGS4 on the 3.8-m UKIRT appear to offer a very efficient way to study the early universe. Such instruments will become even more 
important as the new 8-10-m class telescopes become reality.

Of course, the potential benefits of high-performance infrared arrays in space-based applications are tremendous because of the very low backgrounds which can be achieved with a cryogenic telescope above the atmosphere. Special developments are under way for space-optimised IR array detectors and we will hear about prospects and performance for such instruments as ISOCAM on the ESA Infrared Space Observatory satellite, NICMOS on the NASA Hubble Space Telescope and detectors for the NASA Space Infrared Telescope Facility mission.

Rodger Thompson, Principal Investigator for the Hubble Space Telescope NICMOS project reviewed the performance and characteristics of $\mathrm{HgCdTe}$ arrays and the results of several groundbased imaging programs were presented to illustrate the potential of these devices.

The detectors for the NICMOS instrument are generally referred to as NICMOS 3 detectors by the manufacturer --- Rockwell International. They are HgCdTe photodiodes with a pixel size of $40 \times 40$ microns arranged in a $256 \times 256$ format. The active area of each pixel is about $93 \%$ of the geometrical area and the array is constructed as 4 independent quadrants. One feature of $\mathrm{HgCdTe}$ is that the band gap is chemically tunable; the NICMOS material has a good response from 1.0 2.5 microns. The performance characteristics are as follows:

READ NOISE
DARK CURRENT 25 electrons
QUANTUM EFFICIENCY $\quad$ electron/s @ $65 \%$ K
LINEARITY LIMIT $\quad 200,000$ electrons

Examples of ground-based imagery using NICMOS devices were shown and included the following: S106, NGC7538, IC348, Trapezium: data from McCaughrean, Rayner and Zinnecker. NGC5907, NGC4563: data from University of Hawaii. M87: from M. Rieke. 0156-252, 0406-244: from McCarthy, Persson and West. NGC891: from Thompson and McCaughrean. M3 and M82: Nadeau and Doyon.

Brooke Gregory described the various infrared instruments at NOAO and CTIO. Since about 1986, all of the effort of the IR instrumentation groups at NOAO, in Tucson and in La Serena Chile (Cerro Tololo), has been devoted to building instruments that enable astronomers to exploit some of the several two-dimensional array IR detectors that have become available to the astronomical community.

A graph of the number of "infrared pixels" in service at NOAO as a function of time in years is rather interesting and bears out the remarks about the rapid evolution of this field of astronomy over the past decade. The number of pixels is typically 4000 in 1987-90, rising to 65,000 in 1991 when the first Pt:Si device was used and within the same year jumping to 260,000 as the 4channel SQIID instrument came into operation. The numbers of infrared pixels are still small compared to a Tektronix $2048 \times 2048$ CCD, however.

KPNO and CTIO both provide infrared imagers for all available telescopes including the 4-m telescopes. At KPNO there is a spectrometer (CRSP) with resolving powers of 130 or 700 (2 pixels) and long slit capability. CTIO has a more versatile spectrometer offering resolving powers of $150,700,2000,3000$.

NOAO Tucson is currently working on a system called FIRE - fast readout electronics to solve 3-5 micron co-adding problem. They are also developing a "do-everything" instrument called COB - for Cryogenic Optical Bench - which will contain grisms, LVFs, imaging FP and polarimeter. Finally, an echelle spectrometer with $R=100,000$ is being designed. At CTIO a NICMOS camera has been developed into a "go-anywhere" instrument. It can even be attached to the 4-m 
spectrographs.

Peter Gillingham described IRIS, a new instrumment for the Anglo-Australian telescope using a NICMOS II $128 \times 128$ array. Descriptions of this instrument prior to commissioning have been published in SPIE proceedings and recent astronomical results are shown in the Anglo-Australian Telescope Newsletter. The detector has the properties described by Rodger Thompson.

Jean-Louis Monin reviewed the development of infrared array detectors in France. The work in France involves the following manufacturers and astronomers:

Manufacturers Astronomers

LETI-LIR (CEA CENG) Observatoire de Meudon

$\begin{array}{ll}\text { Ch. Lucas et al. } & \text { D. Rouan et al. }\end{array}$

SOFRADIR C.E. Saclay - Service Astrophysique

J.P. Chatard et al. C. Cesarsky et al.

P.O. Lagage et al.

S.A.T.

Observatoire de Lyon

Y. Carpentier et al. $\quad$ F. Sibille et al.

Applications include:

SPACE MISSIONS: ISOCAM and MARS 94

GROUND-BASED: Imaging (1-17 microns), Adaptive Optics, Interferometry

The ISOCAM instrument will operate from $2.5-17$ microns with pixel sizes of $1.5,3,6$, and 12 "/pixel using $32 \times 32$ arrays (100 microns pitch) in two channels. In the short wavelength channel from 2.5 - 5 microns is an InSb CID device biult by SAT. It has a full well capacity of 4 million electrons and a read noise of 1100 electrons. The long wave detector is built by LETILIR, CEA-CENG and is a 4-17 micron Si:Ga DRO device.

For the MARS 94: Omega project a new $128 \times 1 \mathrm{InSb}$ diode array with a $\mathrm{CCD}$ readout is planned.

For ground-based work a $128 \times 128 \mathrm{HgCdTe}$ array with $\mathrm{CCD}$ readout has been developed by LETI-LIR. The material has a 5.5 micron cutoff, a capacity of 10 million electrons and a read noise of about 400 electrons. Also, a 10 micron camera based on a $64 \times 64 \mathrm{Si}: \mathrm{Ga}$ array derived from ISO has been used successfully.

Results of applications to imaging, adaptive optics and speckle interferometry were presented briefly. New work in progress involves 256x256 HgCdTe/CCD (1992) and 512x512 by 1995 .

Diego Cesarsky gave a brief update on the flight models tests of the ISOCAM infrared imaging instrument for the ESA Infrared Space Observatory. This instrument is an advanced stage of completion and uses the detector technology described above.

In talk No. 7, Judith Pipher presented an overview of infrared arrays for the NASA SIRTF mission and included a discussion of ground-based applications from derivatives of these detectors or precursors to the SIRTF detectors.

Arrays of unprecedented sensitivity are being developed for SIRTF (Space Infrared Telescope Facility), that will span wavelengths from 1.8 - 200 microns. The goal is to be background limited at every wavelength; this goal imposes considerable constraints in the near IR, particularly near 3 microns, where the natural background radiation is a minimum. The near IR arrays should exhibit high quantum efficiency over the 2 - 5.5 micron range, yet also exhibit dark currents < 
$0.03 \mathrm{e} / \mathrm{s}$. In order for a device to not be read noise dominated over integration times of interest, $<10 \mathrm{e}$ is the desired noise level. The devices should be uniform, calibratable, be stable, and relatively immune to charged particle hits (among other properties).

There are 3 SIRTF teams: IRAC (infrared array camera); IRS (infrared spectrometer); MIPS (Multiban infrared photometer system). The first two teams require 256x256 near IR arrays (to 5.5 microns) as well as longer wave arrays. IRAC and IRS both require arrays out to 200 microns. The MIPS detectors are somewhat different, and will not be described here (except where there is overlap in requirements; namely the Ge:Ga IBC for long-wave applications).

IRAC has had the primary responsibility for developing the near IR arrays, and the IRS the midand long-wave arrays. To date, IRAC has contracted SBRC to improve InSb arrays and multiplexers to meet SIRTF requirements. Through doping variations, and geometric variants, as well as processing changes, the new $256 \times 256 \mathrm{InSb}$ arrays are coming closer to meeting SIRTF requirements. Further development in this area is being pursued. Ground-based astronomers also benefit from these developments. In addition, a separate program to develop a low temperature, low noise multiplexer readout (MUX) is being pursued with Valley Oak Semiconductor: Cincinnati Electronics InSb detector arrays (different process) are being bonded to that MUX for initial testing. A comparison between the two vendor's InSb arrays will be conducted this year. IRS has contracted Rockwell to develop Si:As and Si:Sb IBC (Impurity Band Conduction) arrays for the mid-IR. Most of the arrays tested have been bonded to a MUX that is not fully multiplexed; Valley Oak Semiconductor muxes will be developed for these arrays as well. Two alternative vendors (Hughes and Aerojet) have also developed Si:As IBC arrays. IRAC is currently testing the Hughes variety, bonded to the same 58x62 MUX that the InSb test arrays were bonded to. In addition, IRS (with input from the MIPS team), along with JPL (Jet Propulsion Lab) and Rockwell, are developing Ge:Ga IBC arrays for 40-200 micron.

Ground-based applications of SIRTF and SIRTF-like 58x62 InSb array, as well as observations obtained by University of California, San Diego using an Aerojet IBC array. The larger format Rockwell devices will be used on the KAO (Kuiper Airborne Observatory), and the Rochester team hopes to utilize the Ge:Ga IBC long-wave arrays on the KAO in the next few years.

In the next talk, Andy Longmore demonstrated convincingly that infrared array detectors are capable of excellent photometry by presenting many colour-magnitude diagrams of globular and open clusters showing how much more of the lower end of the main sequence could be studied.

Rigaut described observations with an infrared array imaging system capable of near diffractionlimited performance in the near infrared.

Eckart presented an analysis of images of the Galactic Centre obtained with a $62 \times 58 \mathrm{InSb}$ array in the HeI emission line and in the nearby continuum.

Gezari described in detail the operation and performance of a 10 micron camera which uses a 58x62 Si:Ga direct readout array detector manufactured by Hughes/Santa Barbara Research Center The detectors in this array are photoconductors rather than Impurity Band Conduction devices. Many examples of 10 micron imagery were presented.

Finally, Dave Aitken reported the first results of imaging polarimetry in the 10 micron region using an array detector, McLean had earlier shown examples of how well-developed the field of near-infrared imaging polarimetry had become as a result of array detectors. The 10 micron work was done on the UKIRT using the Gezari camera just described which had been adapted for polarimetry, and was a collaboration of groups from the USA (Gezari and McCaughrean), Australia (Aitken and Smith) and the UK (Roche). A complete account of this work will be published elsewhere.

The camera was modified for polarimetry by mounting a cold wire-grid analyser close to the 
Lyot stop within the camera and installing a 10 micron halfwave plate in the UKIRT IRPOL module developed by McLean; the waveplate is at ambient temperature outside of the dewar. A sequence of four rotational settings of the halfwave plate provides four images from which we can derive the Stokes parameters of linear polarization $Q$ and $U$, as well as the total intensity $I$. The percentage linear polarization and position angle of the direction of vibration of the electric vector then follow from conventional formulae.

The primary target for 10 micron polarimetry was the Galactic Center. Determination of polarizations to say $1 \%$ requires that the separate images are photometric to $0.7 \%$ accuracy. In view of the large background in the middle infrared, subject to variability on a time scale of several seconds it is necessary to chop the image; here the chopping secondary was operated at $1 \mathrm{~Hz}$ with a throw of 20 arcsec, sufficient to clear the structure of Sgr A.

A detailed polarization map $60 \times 60$ arcsecs with about 1 arcsec resolution and corresponding to roughly 8 hours of integration shows an intricate polarization structure. The polarization is due to emission from grains aligned by the magnetic field in the filaments of the northem arm and east-west bar with an overlying component of interstellar absorptive polarization.

\section{Conclusions}

Both sessions of the JCM ended with the same feeling, namely, that these two subject areas were in a rapid state of growth and that the astronomical community would continue to hear more and more about their developments throughout the coming decade.

I would like to thank all of the speakers, especially those who kindly provided written summaries of their talks, and I am particulary grateful to Dr. Saul Adelman for his efforts in organising and coordinating Part 1.

\section{References}

Cowie, L.L., Gardner, J.P., Lilly, S.J. and McLean, I.S. 1990, Ap. J. Letters

Elston, R. (ed.) 1991, Astrophysics with Infrared Arrays, Publ. A.S.P. confemece series.

McCaughrean, M.J. 1991, Sky and Telescope, July, 31.

McLean, I.S. 1987, Infrared Astronomy with Arrays, eds. C.G. Wynn-Williams and E.E. Becklin, University of Hawaii.

McLean, I.S., Casali, M.M., Wright, G.S. and Aspin, C. 1989, Proc. 3rd Infrared Detector

Technology Workshop, ed. C. McCreight, NASA Technical Memorandum 102209, 183. 